\title{
Calculation of Weighted Geometric Dilution of Precision
}

\author{
Chien-Sheng Chen, ${ }^{1}$ Yi-Jen Chiu, ${ }^{2}$ Chin-Tan Lee, ${ }^{3}$ and Jium-Ming Lin ${ }^{4}$ \\ ${ }^{1}$ Department of Information Management, Tainan University of Technology, Tainan, Taiwan \\ ${ }^{2}$ Department of Digital Entertainment and Game Design, Taiwan Shoufu University, Tainan, Taiwan \\ ${ }^{3}$ Department of Electronic Engineering, National Quemoy University, Kinmen, Taiwan \\ ${ }^{4}$ Department of Communication Engineering, Chung-Hua University, Hsinchu, Taiwan
}

Correspondence should be addressed to Chien-Sheng Chen; t00243@mail.tut.edu.tw

Received 20 April 2013; Revised 28 August 2013; Accepted 3 September 2013

Academic Editor: Anyi Chen

Copyright (C) 2013 Chien-Sheng Chen et al. This is an open access article distributed under the Creative Commons Attribution License, which permits unrestricted use, distribution, and reproduction in any medium, provided the original work is properly cited.

\begin{abstract}
To achieve high accuracy in wireless positioning systems, both accurate measurements and good geometric relationship between the mobile device and the measurement units are required. Geometric dilution of precision (GDOP) is widely used as a criterion for selecting measurement units, since it represents the geometric effect on the relationship between measurement error and positioning determination error. In the calculation of GDOP value, the maximum volume method does not necessarily guarantee the selection of the optimal four measurement units with minimum GDOP. The conventional matrix inversion method for GDOP calculation demands a large amount of operation and causes high power consumption. To select the subset of the most appropriate location measurement units which give the minimum positioning error, we need to consider not only the GDOP effect but also the error statistics property. In this paper, we employ the weighted GDOP (WGDOP), instead of GDOP, to select measurement units so as to improve the accuracy of location. The handheld global positioning system (GPS) devices and mobile phones with GPS chips can merely provide limited calculation ability and power capacity. Therefore, it is very imperative to obtain WGDOP accurately and efficiently. This paper proposed two formations of WGDOP with less computation when four measurements are available for location purposes. The proposed formulae can reduce the computational complexity required for computing the matrix inversion. The simpler WGDOP formulae for both the 2D and the 3D location estimation, without inverting a matrix, can be applied not only to GPS but also to wireless sensor networks (WSN) and cellular communication systems. Furthermore, the proposed formulae are able to provide precise solution of WGDOP calculation without incurring any approximation error.
\end{abstract}

\section{Introduction}

In positioning the location estimates are determined through the received signals transmitted by the mobile devices at a set of base stations (BSs), satellites, or other sensors. First, the length or direction of the radio path is determined through signal measurements. Secondly, the MS position is derived from radio location algorithms and known geometric relationships. Mobile positioning systems have received significant attention, and various location technologies have been proposed in the past few years. Among the techniques for mobile positioning there are two major categories-handsetbased and network-based schemes. Both approaches have their advantages and limitations. Global positioning system
(GPS) is a positioning system that can provide position, velocity, and time information to a user. Handset-based solutions generally require a handset modification to calculate its own position when they are fully or partially equipped with a GPS receiver. The advantages of using handsetbased methods are that they have global coverage and usually provide much more accurate location measurements. The drawbacks of the handset-based methods include cost, redundant hardware, and economical integrated technology. The reliability of GPS measurements is greatly compromised in a building or shadowed environments, where direct lineof-sight (LOS) propagation is not available. Without the aid of satellite systems, network-based positioning schemes use time and angle measurements to determine the MS location 
or to assist the process of MS location determination. Instead of using all seven BSs, four BSs with better geometry are good enough to provide sufficient measurements for positioning in cellular communication networks. The network-based location schemes are relatively less complex on hardware when compared with the handset-based methods. They can be employed in many situations where GPS signal cannot, for example, indoor environment and urban canyon areas, or when GPS-embedded handsets are not available. For many applications in wireless sensor networks (WSN), like environmental sensing and activities measuring, it is crucial to know the locations of the sensor nodes in network-based positioning; this is known as a "localization problem" [1]. An ideal location technology should be able to provide a robust estimate of location in all environments.

This paper considers both the network-based method and the handset-based method, employing the concept of geometric dilution of precision (GDOP), which was initially developed as a criterion for selecting the optimal $3 \mathrm{D}$ geometric configuration of satellites in GPS. The general object of the GPS satellite selection algorithm is to minimize the GDOP to improve the position accuracy. The smaller value of GDOP is calculated, the better geometric configuration we will have. The redundant measurements will bring large amount of computation and may not provide significantly improved location accuracy. When enough measurements are available, the optimal measurements selected with the minimum GDOP can prevent the poor geometry effects and have the potential of obtaining greater location accuracy.

There have been extensive researches trying to obtain an approximate GDOP value without executing matrix inversion in the past few years. Simon and El-Sherief $[2,3]$ proposed the employment of back-propagation neural network (BPNN) [4] to obtain an approximation for the GDOP function. The BPNN is employed to learn the relationship between the entries of a measurement matrix and the eigenvalues of its inverse. Three other input-output relationships were proposed in [5]. We present the resilient back-propagation (Rprop) architectures to obtain the approximate GDOP [6]. The matrix inversion method for GDOP calculation is born with significant computational burden. GDOP is approximately inversely proportional to the volume of the tetrahedron formed by the tips of four unit vectors directed to the selected satellites in GPS [7]. The four satellites evenly distribute with the maximum volume which brings the more accurate location estimation. The maximum volume method requires low computing time in selecting a subset with the largest tetrahedron as the optimum [8]. However, it is not suitable to use this method because it may not select the desired satellites with the minimum GDOP. The main disadvantage of these methods is to incur approximation errors. To avoid these disadvantages, a simple closed-form formula for GDOP calculation is proposed in [9].

Traditionally, the GDOP computation assumes that the pseudorange errors are independent and identical [10]. Several methods based on GDOP have been proposed to improve the GPS positioning accuracy $[7,9,11]$. In fact, measurements usually have different error variances [12]. Ranging error of GPS is caused by many sources, such as the effect of ionosphere delay, tropospheric delay, carrierto-noise ratio, and multipath. GDOP and the effect of these errors can be considered simultaneously; the extension of GDOP criteria is used for satellite selection in [13]. The satellite signal is also approximated by combining the user range accuracy value, carrier-to-noise ratio, elevation angle, and the date of ephemeris. The weighted GDOP (WGDOP) which takes these errors into account was proposed in [14]. The elevation of each satellite and signal-to-noise-ratio (SNR) are introduced as fuzzy subset to weight GDOP and provide the positioning solution [15]. When baro-altitude measurements or a priori terrain elevation information is used, the conventional GDOP formula cannot be applied and must be modified [16] in order to reduce the influence of satellites with a large error and evaluates the influence of each satellite on the arrangement of satellites. The GDOP was focused as a factor to determine the weight matrix and improve precision in GPS measurements [17]. The combinations of the GPS and Galileo satellite constellations will provide more visible satellites with better geometric distribution, and the availability of satellites will be significantly improved. A novel algorithm, namely, the WGDOP minimum algorithm, was proposed in [18] for the combined GPS-Galileo navigation receiver. In addition to the aforementioned, several papers which focus on WGDOP concepts have been proposed to improve the GPS positioning accuracy [19-21]. Taking the different variances of the satellites into account, researchers have proposed various WGDOP measures [13-21]. Much of the research literature needs matrix inversion to calculate WGDOP. Though they can guarantee to achieve the optimal subset, the computational complexity is usually too expensive to be practical.

High accuracy in wireless positioning system requires both the accurate measurement and a good effect of GDOP. When the measurements have different error variances or come from integrated positioning systems, WGDOP minimum criterion is appropriate to select the appropriate measurement units to diminish the positioning error. The optimal measurements selected with the minimum WGDOP can help reduce the adverse geometry effects. Increasing the number of satellites will always reduce the WGDOP value, since the best WGDOP can be obtained by computing all satellites in view. If the number of visible satellites is not large, the all-in-view method is a good choice to provide high accuracy positioning [15]. In order to further improve the positioning accuracy, the combined use of multiconstellation can be employed. There will be 70 90 navigation satellites operating at the same time when Glonass and Galileo reach full operation capability [22]. In any moment, there are more than 30 satellites in view in the multiconstellation navigation systems. To employ all-in-view method for positioning is very difficult for us in the future. Due to limited resources associated with many mobile devices and because the number of visible satellites is very large [18], measurement unit selection techniques can be used. If we select 4 out of 30 satellites, the number of possible subset is 27405 . The calculation of WGDOP is a time and power consuming process, and fast calculation of WGDOP is most anticipated. WGDOP is computed for all subsets, and 
the subset which gives the smallest WGDOP is selected for location estimation.

The growth of GPS embedded into current mobile phones continues to grow rapidly, as many mobile phones now are already equipped with GPS inside. Despite their performance increases, these devices still possess limited resources, such as the number of channels, battery capacities, and processing capability. Satellite selection can reduce the number of satellites used to position and as a result reducing the amount of calculation greatly. The number of measurements can be restricted and the resulting saving in load on the processor can be used to offer more spare processing time which can be used for other user specific requirements. On the other hand, reducing the signal-processing time of the receiver dedicated to satellite selection implies both increasing the processing capabilities available for other purposes and saving battery. The conventional method for calculating WGDOP is to use matrix inversion, which requires enormous amount of computation. This can present challenges to real-time practical applications. Therefore, it is very critical to select a subset with the most appropriate measurement units rapidly and reasonably before positioning.

To calculate WGDOP in the form of $2 \mathrm{D}$ and $3 \mathrm{D}$ formulations effectively, the closed-form solutions for two WGDOP formations are proposed for the case of each measurement with a unique variance and one of the measurements with higher location precision. The computation load of the proposed formulae is greatly less than that of the matrix inversion method. When exactly four measurements are used, the proposed formulae provide the best computational efficiency. The proposed formulae can also provide the exact solution to the WGDOP calculation and do not incur any approximation errors. The relatively simple closed-form WGDOP formulae can be implemented in the aforementioned papers [1321]. The calculations of WGDOP for fast evaluation can be applied in GPS, WSN, and cellular communication systems. In practice, the measurement units of GPS, WSN, and cellular communication systems are satellites, sensors, and BSs, respectively.

The author of this paper proposed two novel architectures and presented four original architectures based on Rprop neural network to approximate WGDOP [23]. The disadvantage of Rprop-based WGDOP algorithm is the need of a training phase with several input-output patterns. We collect the elements of related matrix and the desired WGDOP value to train the neural network prior to the practicaluse. After the training, the elements of geometry matrix and weighted matrix as input data can not only pass through the trained Rprop but also predict the better appropriate WGDOP. From simulation results, the proposed WGDOP formulae always provide much better accuracy than Rprop-based WGDOP approximation [23]. But the proposed efficient formulae for WGDOP have been developed when there are exactly four measurement units used.

The remainder of this paper is organized as follows: Section 2 describes the concepts of GDOP and WGDOP. Section 3 reviews an efficient solution for the calculation of GDOP. The closed-form formulae for WGDOP calculations in the case of four measurements with unequal variances are proposed in Section 4. In Section 5, we examine the performance of the proposed formulae through simulation experiments. Conclusion is given in Section 6.

\section{GDOP and WGDOP}

GDOP is a task of choosing the appropriate measurement units, which results in a better geometric configuration and a more accurate position estimate. In order to achieve better positioning accuracy, it is desirable to select the combination of measurements with GDOP as small as possible. Using a 3D Cartesian coordinate system, the distances between satellite $i$ and the user can be expressed as

$$
r_{i}=\sqrt{\left(x-X_{i}\right)^{2}+\left(y-Y_{i}\right)^{2}+\left(z-Z_{i}\right)^{2}}+C \cdot t_{b}+v_{r i},
$$

where $(x, y, z)$ and $\left(X_{i}, Y_{i}, Z_{i}\right)$ are the locations of the user and satellite $i$, respectively; $C$ is the speed of light, $t_{b}$ denotes the time offset, and $v_{r i}$ is pseudorange measurements noise. Equation (1) is linearized through the use of a Taylor series expansion around the approximate user position $(\widehat{x}, \widehat{y}, \widehat{z})$ and the first two terms are retained. Defining $\widehat{r}_{i}$ as $r_{i}$ at $(\widehat{x}, \widehat{y}, \widehat{z})$, we can obtain

$$
\Delta r=r_{i}-\widehat{r}_{i} \cong e_{i 1} \delta_{x}+e_{i 2} \delta_{y}+e_{i 3} \delta_{z}+C \cdot t_{b}+v_{r i},
$$

where $\delta_{x}, \delta_{y}$, and $\delta_{z}$ are, respectively, coordinate offsets of $x$, $y$, and $z$,

$$
\begin{gathered}
e_{i 1}=\frac{\widehat{x}-X_{i}}{\widehat{r}_{i}}, \quad e_{i 2}=\frac{\widehat{y}-Y_{i}}{\widehat{r}_{i}}, \quad e_{i 3}=\frac{\widehat{z}-Z_{i}}{\widehat{r}_{i}}, \\
\hat{r}_{i}=\sqrt{\left(\widehat{x}-X_{i}\right)^{2}+\left(\hat{y}-Y_{i}\right)^{2}+\left(\widehat{z}-Z_{i}\right)^{2}} .
\end{gathered}
$$

$\left(e_{i 1}, e_{i 2}, e_{i 3}\right), i=1,2, \ldots, n$, denote the line-of-sight (LOS) vector from the satellites to the user.

The linearized pseudorange measurement equations take the form

$$
z=H \delta+v,
$$

where $z=\left[\begin{array}{c}r_{1}-\widehat{r}_{1} \\ r_{2}-\widehat{r}_{2} \\ \vdots \\ r_{n}-\widehat{r}_{n}\end{array}\right], \delta=\left[\begin{array}{c}\delta_{x} \\ \delta_{y} \\ \delta_{z} \\ c \cdot t_{b}\end{array}\right], v=\left[\begin{array}{c}v_{r_{1}} \\ v_{r 2} \\ \vdots \\ v_{r n}\end{array}\right]$, and $H=$ $\left[\begin{array}{cccc}e_{11} & e_{12} & e_{13} & 1 \\ e_{21} & e_{22} & e_{23} & 1 \\ \vdots & \vdots & \vdots & \vdots \\ e_{n 1} & e_{n 2} & e_{n 3} & 1\end{array}\right]$ is the geometry matrix.

According to the least square algorithm (LS), the solution to (4) is given by

$$
\widehat{\delta}=\left(H^{T} H\right)^{-1} H z .
$$

Assume that the pseudorange errors are uncorrelated with equal variances $\sigma^{2}$, the error covariance matrix can be expressed as

$$
E\left[(\widehat{\delta}-\delta)(\widehat{\delta}-\delta)^{T}\right]=\sigma^{2} \cdot\left(H^{T} H\right)^{-1} .
$$


The variances are functions of the diagonal elements of $\left(H^{T} H\right)^{-1}$. The GDOP is a measure of accuracy for positioning systems and depends solely on the geometry matrix $H$

$$
\mathrm{GDOP}=\sqrt{\operatorname{tr}\left(H^{T} H\right)^{-1}}
$$

In fact, each measurement error does not have the same variance, especially for the combination of different systems. The covariance matrix represents the uncertainty in the pseudorange measurements and has the following form:

$$
E\left(v v^{T}\right)=\left[\begin{array}{ccccc}
\sigma_{1}^{2} & 0 & 0 & 0 & 0 \\
0 & \sigma_{2}^{2} & 0 & 0 & 0 \\
0 & 0 & \sigma_{3}^{2} & 0 & 0 \\
0 & 0 & 0 & \ddots & 0 \\
0 & 0 & 0 & 0 & \sigma_{n}^{2}
\end{array}\right]
$$

$W$ is a diagonal matrix and defined as a weighted matrix

$$
W=\left[\begin{array}{ccccc}
1 / \sigma_{1}^{2} & 0 & 0 & 0 & 0 \\
0 & 1 / \sigma_{2}^{2} & 0 & 0 & 0 \\
0 & 0 & 1 / \sigma_{3}^{2} & 0 & 0 \\
0 & 0 & 0 & \ddots & 0 \\
0 & 0 & 0 & 0 & 1 / \sigma_{n}^{2}
\end{array}\right]=\left[\begin{array}{ccccc}
k_{1} & 0 & 0 & 0 & 0 \\
0 & k_{2} & 0 & 0 & 0 \\
0 & 0 & k_{3} & 0 & 0 \\
0 & 0 & 0 & \ddots & 0 \\
0 & 0 & 0 & 0 & k_{n}
\end{array}\right],
$$

where $\sigma_{i}^{2}=1 / k_{i}, i=1,2, \ldots, n$ are the variances of the measurement errors.

With the weighting matrix defined above, the solution to the weighted least square (WLS) can be expressed as

$$
\widehat{\delta}=\left(H^{T} W H\right)^{-1} H^{T} W z .
$$

Taking into account that all measurement units contain different variances, the positioning algorithm using WLS estimation provides higher location accuracy than the LS estimation. Having considered both the geometric configuration and the priori knowledge of error models simultaneously, we choose WGDOP, instead of GDOP, for measurements selection to achieve effective performance improvement. The optimal subset is the one with the minimum WGDOP, which is given by the trace of the inverse of the $H^{T} W H$ matrix

$$
\text { WGDOP }=\sqrt{\operatorname{tr}\left(H^{T} W H\right)^{-1}}
$$

We can compute the WGDOP value of each subset, and then the subsets with minimum WGDOP are the selected measurement units. The conventional method for calculating WGDOP is to use matrix inversion for all subsets. The method can guarantee the optimal subset; however, it presents a heavy computational burden.

\section{Calculation of GDOP for Four Measurements}

In the time of arrival (TOA) positioning methods, which is applied to GPS, the TOA circle becomes the sphere in space and the fourth measurement is required to solve the receiver-clock bias for a $3 \mathrm{D}$ solution. The bias is the clock synchronization error between the receiver and the satellite. In practice, the time of user is significantly more inaccurate than that of an atomic clock on the satellite. In order to correct the clock bias errors present at the receiver of the users end, the measurement from the fourth satellite is employed. Getting information from the fourth measurement makes it possible to solve for this fourth unknown. Even though there are more than four satellites in view, a subset with four satellites is sufficient providing the sufficient measurements for navigation solution even though more than four satellites are in view, which is called the optimum four GPS satellites positioning [15]. The selection of four visible satellites to provide the suitable GPS positioning accuracy is presented in several papers [13-17]. Thus, we propose to take only four BSs with better geometry out of seven to estimate the MS location in cellular communication networks. For practical real-time applications, the number of selected measurement units should not be large. The efficient closed-form solution with simpler calculation for a four-satellite case is proposed in [9].

By using of the following properties:

$$
\begin{aligned}
& (U V)^{-1}=V^{-1} U^{-1}, \\
& \operatorname{tr}(U V)=\operatorname{tr}(V U),
\end{aligned}
$$

we have $\operatorname{tr}(U V)^{-1}=\operatorname{tr}(V U)^{-1}$.

From (7), the GDOP can be written as

$$
\text { GDOP }=\sqrt{\operatorname{tr}\left(H^{T} H\right)^{-1}}=\sqrt{\operatorname{tr}\left(H H^{T}\right)^{-1}} .
$$

By defining the variable

$$
B_{i j}=e_{i 1} e_{j 1}+e_{i 2} e_{j 2}+e_{i 3} e_{j 3}+1, \quad 1 \leq i<j \leq 4,
$$

and using the following relation that

$$
e_{i 1}^{2}+e_{i 2}^{2}+e_{i 3}^{2}=1, \quad i=1,2,3,4,
$$

we have

$$
H H^{T}=\left[\begin{array}{cccc}
2 & B_{12} & B_{13} & B_{14} \\
B_{12} & 2 & B_{23} & B_{24} \\
B_{13} & B_{23} & 2 & B_{34} \\
B_{14} & B_{24} & B_{34} & 2
\end{array}\right]
$$

Defining the following variables:

$$
\begin{gathered}
a=\left(B_{12} B_{34}+B_{13} B_{24}-B_{14} B_{23}\right)^{2}-4 B_{12} B_{34} B_{13} B_{24}, \\
b=16-4\left(B_{12}^{2}+B_{13}^{2}+B_{14}^{2}+B_{23}^{2}+B_{24}^{2}+B_{34}^{2}\right), \\
c=2\left[B_{12}\left(B_{13} B_{23}+B_{14} B_{24}\right)+B_{34}\left(B_{13} B_{14}+B_{23} B_{24}\right)\right],
\end{gathered}
$$

the GDOP can be written as [9]

$$
\text { GDOP }=\sqrt{\frac{16+b+c}{a+b+2 c}} .
$$

Note that both $B_{12} B_{34}$ and $B_{13} B_{24}$ appear twice in the expression of $a$, and two multiplications can be eliminated. The closed-form equation needs only 39 multiplications, 34 additions, 1 division, and 1 square root. 


\section{Calculation of WGDOP for Four Measurements}

To further reduce the computational overhead and improve the location performance, the selection of the optimal measurement units is necessary. Since the statistics of different location measurement units are, in general, not equal to each other, WGDOP is appropriate to an index for the precision of location in different networks, such as GPS, WSN, and cellular communication systems. The steps for positioning are listed as follows.

(1) We will first select four measurements among $n$ measurement units to generate the subsets; thus, the $n$ measurement units are classified into $C(n, 4)$ possible subsets.

(2) WGDOP is computed for all possible subsets of four measurement units.

(3) The subset which gives the smallest WGDOP is selected as the optimal subset.

(4) Finally, the four measurements of this subset can be used to find out the location solution.

The calculation of WGDOP takes considerable computing time; it is very imperative to accelerate the computation of WGDOP in real-time application. In this paper, we propose the efficient closed-form solution of two WGDOP formations, which includes the effect of GDOP and error statistics properties simultaneously. These solutions, with the simplified form for WGDOP calculation, can apply to all possible subsets in $3 \mathrm{D}$ and $2 \mathrm{D}$ scenarios and require much less computation compared to the conventional matrix inversion method.

\subsection{Type 1: Four Measurements Have Different Error Variances}

4.1.1. 3D Case. From (11) and by using the properties of the basic algebra theory, WGDOP can be alternatively recognized as

$$
\text { WGDOP }=\sqrt{\operatorname{tr}\left(H^{T} W H\right)^{-1}}=\sqrt{\operatorname{tr}\left(H H^{T} W\right)^{-1}} .
$$

By using (14) and (15), we have

$$
\begin{aligned}
H H^{T} W= & {\left[\begin{array}{llll}
e_{11} & e_{12} & e_{13} & 1 \\
e_{21} & e_{22} & e_{23} & 1 \\
e_{31} & e_{32} & e_{33} & 1 \\
e_{41} & e_{42} & e_{43} & 1
\end{array}\right]\left[\begin{array}{cccc}
e_{11} & e_{21} & e_{31} & e_{41} \\
e_{12} & e_{22} & e_{32} & e_{42} \\
e_{13} & e_{23} & e_{33} & e_{43} \\
1 & 1 & 1 & 1
\end{array}\right] } \\
& \times\left[\begin{array}{cccc}
k_{1} & 0 & 0 & 0 \\
0 & k_{2} & 0 & 0 \\
0 & 0 & k_{3} & 0 \\
0 & 0 & 0 & k_{4}
\end{array}\right]
\end{aligned}
$$

thus

$$
H H^{T} W=\left[\begin{array}{cccc}
2 k_{1} & k_{2} B_{12} & k_{3} B_{13} & k_{4} B_{14} \\
k_{1} B_{12} & 2 k_{2} & k_{3} B_{23} & k_{4} B_{24} \\
k_{1} B_{13} & k_{2} B_{23} & 2 k_{3} & k_{4} B_{34} \\
k_{1} B_{14} & k_{2} B_{24} & k_{3} B_{34} & 2 k_{4}
\end{array}\right] .
$$

The WGDOP parameter is the square root of the sum of diagonal terms of the matrix $\left(H H^{T} W\right)^{-1}$

WGDOP

$$
\begin{aligned}
& =\sqrt{\operatorname{tr}\left(H H^{T} W\right)^{-1}} \\
& =\sqrt{\left(H H^{T} W\right)_{1,1}^{-1}+\left(H H^{T} W\right)_{2,2}^{-1}+\left(H H^{T} W\right)_{3,3}^{-1}+\left(H H^{T} W\right)_{4,4}^{-1}}
\end{aligned}
$$

$\left(H H^{T} W\right)_{i, i}^{-1}$ is defined as the $i$ th element on the diagonal of matrix $\left(H H^{T} W\right)^{-1}$

$$
\begin{aligned}
\operatorname{tr}\left(H H^{T} W\right)^{-1} & =\sum_{i=1}^{4}\left(H H^{T} W\right)_{i, i}^{-1} \\
& =\frac{\operatorname{tr}\left[\operatorname{adj}\left(H H^{T} W\right)\right]}{\operatorname{det}\left(H H^{T} W\right)}=\sum_{i=1}^{4} \frac{\operatorname{cof}_{i, i}\left(H H^{T} W\right)}{\operatorname{det}\left(H H^{T} W\right)} .
\end{aligned}
$$

The term $\operatorname{adj}\left(H H^{T} W\right)$ is the adjoint of $H H^{T} W$ and the cofactor, and $\operatorname{cof}_{i, i}\left(H H^{T} W\right)$ is the determinant of the submatrix of $H H^{T} W$ by deleting the $i$ th row and the $i$ th column. The cofactors can be obtained as

$$
\begin{aligned}
& \operatorname{cof}_{1,1}\left(H H^{T} W\right) \\
& \quad=k_{2} k_{3} k_{4}\left[8+2\left(B_{23} B_{24} B_{34}-\left(B_{23}^{2}+B_{24}^{2}+B_{34}^{2}\right)\right)\right],
\end{aligned}
$$

$$
\begin{aligned}
& \operatorname{cof}_{2,2}\left(H H^{T} W\right) \\
& \quad=k_{1} k_{3} k_{4}\left[8+2\left(B_{13} B_{14} B_{34}-\left(B_{13}^{2}+B_{14}^{2}+B_{34}^{2}\right)\right)\right],
\end{aligned}
$$

$$
\begin{aligned}
& \operatorname{cof}_{3,3}\left(H H^{T} W\right) \\
& \quad=k_{1} k_{2} k_{4}\left[8+2\left(B_{12} B_{14} B_{24}-\left(B_{12}^{2}+B_{14}^{2}+B_{24}^{2}\right)\right)\right]
\end{aligned}
$$

$$
\begin{aligned}
& \operatorname{cof}_{4,4}\left(H H^{T} W\right) \\
& \quad=k_{1} k_{2} k_{3}\left[8+2\left(B_{12} B_{13} B_{23}-\left(B_{12}^{2}+B_{13}^{2}+B_{23}^{2}\right)\right)\right] .
\end{aligned}
$$

After some algebraic manipulation, the determinant of matrix $H H^{T} W$ can be written as

$$
\begin{aligned}
\operatorname{det}\left(H H^{T} W\right)= & k_{1} k_{2} k_{3} k_{4} \\
\times & \left\{16+2\left[B_{23} B_{24} B_{34}-\left(B_{23}^{2}+B_{24}^{2}+B_{34}^{2}\right)\right]\right. \\
& +2\left[B_{13} B_{14} B_{34}-\left(B_{13}^{2}+B_{14}^{2}+B_{34}^{2}\right)\right]
\end{aligned}
$$




$$
\begin{gathered}
+2\left[B_{12} B_{14} B_{24}-\left(B_{12}^{2}+B_{14}^{2}+B_{24}^{2}\right)\right] \\
+2\left[B_{12} B_{13} B_{23}-\left(B_{12}^{2}+B_{13}^{2}+B_{23}^{2}\right)\right] \\
+\left(B_{12} B_{34}+B_{13} B_{24}-B_{14} B_{23}\right)^{2} \\
-4 B_{12} B_{34} B_{13} B_{24} \\
+2\left[B_{12}\left(B_{13} B_{23}+B_{14} B_{24}\right)\right. \\
\left.\left.+B_{34}\left(B_{13} B_{14}+B_{23} B_{24}\right)\right]\right\} .
\end{gathered}
$$

Defining the following variables:

$$
\begin{aligned}
& p=\left[B_{23} B_{24} B_{34}-\left(B_{23}^{2}+B_{24}^{2}+B_{34}^{2}\right)\right], \\
& q=\left[B_{13} B_{14} B_{34}-\left(B_{13}^{2}+B_{14}^{2}+B_{34}^{2}\right)\right], \\
& m=\left[B_{12} B_{14} B_{24}-\left(B_{12}^{2}+B_{14}^{2}+B_{24}^{2}\right)\right], \\
& n=\left[B_{12} B_{13} B_{23}-\left(B_{12}^{2}+B_{13}^{2}+B_{23}^{2}\right)\right],
\end{aligned}
$$

then we have

$$
\mathrm{WGDOP}=\sqrt{\frac{2 \cdot\left[\left(1 / k_{1}\right) \cdot(4+p)+\left(1 / k_{2}\right) \cdot(4+q)+\left(1 / k_{3}\right) \cdot(4+m)+\left(1 / k_{4}\right) \cdot(4+n)\right]}{a+c-16+2 \cdot[(4+p)+(4+q)+(4+m)+(4+n)]}} .
$$

When four measurements have different error variances, the closed-form solution for WGDOP is given by

\section{WGDOP}

$$
=\sqrt{\frac{2 \cdot\left(\left(1 / k_{1}\right) \cdot P+\left(1 / k_{2}\right) \cdot Q+\left(1 / k_{3}\right) \cdot M+\left(1 / k_{4}\right) \cdot N\right)}{a+c-16+2 \cdot(P+Q+M+N)}},
$$

where $P=4+p, Q=4+q, M=4+m, N=4+n$.

Note that $B_{12} B_{34}, B_{13} B_{24}, B_{12} B_{13} B_{23}, B_{12} B_{14} B_{24}$, $B_{13} B_{14} B_{34}, B_{23} B_{24} B_{34}, B_{12}^{2}, B_{13}^{2}, B_{14}^{2}, B_{23}^{2}, B_{24}^{2}, B_{34}^{2},(4+p)$, $(4+q),(4+m)$, and $(4+n)$ all appear twice in the express for WGDOP; thus sixteen multiplications and four additions can be eliminated. The values of $1 / k_{i}, i=1,2,3,4$, are assumed to be already known before the calculation of (28); thus they can be treated as constants. From Table 1, the closed-form equation needs only 42 multiplications (including the constant multiplications by 4, 2, 2, and 2), 48 additions, 1 division, and 1 square root. Due to many parameters in the numerator and the denominator of (27) simultaneously, the computational complexity of the proposed criteria can be reduced.

4.1.2. 2 D Case. From algebra theory, we know that solving the four unknowns requires at least four independent equations. When three measurements are utilized to determine the user location, a $2 \mathrm{D}$ position solution is obtained. This means that at least three measurements are required to determine the $2 \mathrm{D}$ position of the users. The complexity of computing the inverse of a $3 \times 3$ square matrix is very low. When four measurements are available for the $2 \mathrm{D}$ scenarios, we propose the simple closed-form formulae of the WGDOP calculations. The geometry matrix which is composed of four location measurement units in $2 \mathrm{D}$ environments is

$$
H=\left[\begin{array}{lll}
e_{11} & e_{12} & 1 \\
e_{21} & e_{22} & 1 \\
e_{31} & e_{32} & 1 \\
e_{41} & e_{42} & 1
\end{array}\right]
$$

where $e_{i 1}=\left(\widehat{x}-X_{i}\right) / \widehat{r}_{i}, e_{i 2}=\left(\widehat{y}-Y_{i}\right) / \widehat{r}_{i}$, and $\widehat{r}_{i}=$ $\sqrt{\left(\widehat{x}-X_{i}\right)^{2}+\left(\widehat{y}-Y_{i}\right)^{2}}, i=1,2,3,4$.

Denoting

$$
B_{i j}=e_{i 1} e_{j 1}+e_{i 2} e_{j 2}+1, \quad 1 \leq i<j \leq 4,
$$

and using the fact that

$$
e_{i 1}^{2}+e_{i 2}^{2}=1
$$

WGDOP in the $2 \mathrm{D}$ case can be expressed as (28). The difference between the $2 \mathrm{D}$ and $3 \mathrm{D}$ scenarios of WGDOP calculation is in the calculation of $B_{i j}, 1 \leq i<j \leq 4$. The computational complexity in the $2 \mathrm{D}$ case is 6 multiplications and 6 additions fewer than that in the $3 \mathrm{D}$ case. Therefore, the closed-form equation needs only 36 multiplications (including the constant multiplications by 4, 2, 2, and 2), 42 additions, 1 division, and 1 square root.

\subsection{Type 2: Four Measurements Have Two Types of Error Var- iances}

4.2.1. 3D Case. In the case of one measurement gives better accuracy than the others, the closed-form solution for WGDOP formulation is proposed here. The situation may occur in some positioning systems. For example, the BS serving a particular MS is called the serving BS, which provides the more accurate measurements in cellular communication systems [24]. Assume that the measurement variances of the serving BS and nonserving BSs are $\sigma_{1}^{2}$ and $\sigma_{2}^{2}$, respectively. Regarding the two types of the error variances, the weight matrix should be modified as follows:

$$
W=\left[\begin{array}{cccc}
1 / \sigma_{1}^{2} & 0 & 0 & 0 \\
0 & 1 / \sigma_{2}^{2} & 0 & 0 \\
0 & 0 & 1 / \sigma_{2}^{2} & 0 \\
0 & 0 & 0 & 1 / \sigma_{2}^{2}
\end{array}\right]=\left[\begin{array}{cccc}
\omega & 0 & 0 & 0 \\
0 & 1 & 0 & 0 \\
0 & 0 & 1 & 0 \\
0 & 0 & 0 & 1
\end{array}\right],
$$

where $\omega$ is the ratio of the nonserving BS error variance to the serving BS error variance:

$$
\omega=\frac{\sigma_{2}^{2}}{\sigma_{1}^{2}}
$$


TABLE 1: The complexity of WGDOP calculation when the four measurements have different error variances.

\begin{tabular}{lcccc}
\hline & Multiplications & Additions & Division & $\begin{array}{c}\text { Square } \\
\text { root }\end{array}$ \\
\hline$B_{12}$ & 3 & 3 & 0 & 0 \\
$B_{13}$ & 3 & 3 & 0 & 0 \\
$B_{14}$ & 3 & 3 & 0 & 0 \\
$B_{23}$ & 3 & 3 & 0 & 0 \\
$B_{24}$ & 3 & 3 & 0 & 0 \\
$B_{34}$ & 3 & 3 & 0 & 0 \\
$a$ & 6 & 3 & 0 & 0 \\
$c$ & 7 & 3 & 0 & 0 \\
$p$ & 3 & 3 & 0 & 0 \\
$q$ & 2 & 3 & 0 & 0 \\
$m$ & 1 & 3 & 0 & 0 \\
$n$ & 0 & 3 & 0 & 0 \\
WGDOP & 5 & 7 & 0 & 0 \\
(numerator) & & & & \\
WGDOP & 0 & 5 & 0 & 0 \\
(denominator) & & 0 & 1 & 1 \\
WGDOP & 42 & 48 & 1 & 1 \\
\hline Total & & & &
\end{tabular}

TABLE 2: The complexity of WGDOP calculation when the four measurements have two types of error variances.

\begin{tabular}{lcccc}
\hline & Multiplications & Additions & Division & $\begin{array}{c}\text { Square } \\
\text { root }\end{array}$ \\
\hline$B_{12}$ & 3 & 3 & 0 & 0 \\
$B_{13}$ & 3 & 3 & 0 & 0 \\
$B_{14}$ & 3 & 3 & 0 & 0 \\
$B_{23}$ & 3 & 3 & 0 & 0 \\
$B_{24}$ & 3 & 3 & 0 & 0 \\
$B_{34}$ & 3 & 3 & 0 & 0 \\
$a$ & 6 & 3 & 0 & 0 \\
$c$ & 7 & 3 & 0 & 0 \\
$p$ & 3 & 3 & 0 & 0 \\
$q$ & 2 & 3 & 0 & 0 \\
$m$ & 1 & 3 & 0 & 0 \\
$n$ & 0 & 3 & 0 & 0 \\
WGDOP & 2 & 5 & 0 & 0 \\
(numerator) & & & & \\
WGDOP & 0 & 3 & 0 & 0 \\
(denominator) & & 0 & 1 & 1 \\
WGDOP & 39 & 44 & 1 & 1 \\
\hline Total & & & & \\
\hline & & & & 0
\end{tabular}

In this case, we have

$$
H H^{T} W=\left[\begin{array}{cccc}
2 \omega & B_{12} & B_{13} & B_{14} \\
\omega B_{12} & 2 & B_{23} & B_{24} \\
\omega B_{13} & B_{23} & 2 & B_{34} \\
\omega B_{14} & B_{24} & B_{34} & 2
\end{array}\right],
$$

and the cofactors can be obtained to be

$$
\begin{aligned}
& \operatorname{cof}_{1,1}\left(H H^{T} W\right) \\
& \quad=\left[8+2\left(B_{23} B_{24} B_{34}-\left(B_{23}^{2}+B_{24}^{2}+B_{34}^{2}\right)\right)\right], \\
& \operatorname{cof}_{2,2}\left(H H^{T} W\right) \\
& \quad=\omega\left[8+2\left(B_{13} B_{14} B_{34}-\left(B_{13}^{2}+B_{14}^{2}+B_{34}^{2}\right)\right)\right], \\
& \operatorname{cof}_{3,3}\left(H H^{T} W\right) \\
& \quad=\omega\left[8+2\left(B_{12} B_{14} B_{24}-\left(B_{12}^{2}+B_{14}^{2}+B_{24}^{2}\right)\right)\right], \\
& \operatorname{cof}_{4,4}\left(H H^{T} W\right) \\
& \quad=\omega\left[8+2\left(B_{12} B_{13} B_{23}-\left(B_{12}^{2}+B_{13}^{2}+B_{23}^{2}\right)\right)\right] .
\end{aligned}
$$

The determinants of matrix $H H^{T} W$ are found to be

$$
\begin{aligned}
\operatorname{det}\left(H H^{T} W\right)=\omega & \left\{16+2\left[B_{23} B_{24} B_{34}-\left(B_{23}^{2}+B_{24}^{2}+B_{34}^{2}\right)\right]\right. \\
+ & 2\left[B_{13} B_{14} B_{34}-\left(B_{13}^{2}+B_{14}^{2}+B_{34}^{2}\right)\right] \\
+ & 2\left[B_{12} B_{14} B_{24}-\left(B_{12}^{2}+B_{14}^{2}+B_{24}^{2}\right)\right] \\
+ & 2\left[B_{12} B_{13} B_{23}-\left(B_{12}^{2}+B_{13}^{2}+B_{23}^{2}\right)\right] \\
+ & \left(B_{12} B_{34}+B_{13} B_{24}-B_{14} B_{23}\right)^{2} \\
& -4 B_{12} B_{34} B_{13} B_{24} \\
+ & 2\left[B_{12}\left(B_{13} B_{23}+B_{14} B_{24}\right)\right. \\
& \left.\left.+B_{34}\left(B_{13} B_{14}+B_{23} B_{24}\right)\right]\right\},
\end{aligned}
$$

and we have

WGDOP

$$
=\sqrt{\frac{2 \cdot[(1 / \omega) \cdot(4+p)+(4+q)+(4+m)+(4+n)]}{a+c-16+2 \cdot[(4+p)+(4+q)+(4+m)+(4+n)]}} .
$$

The closed-form WGDOP for the case of exactly four measurements can be expressed as

$$
\begin{aligned}
\mathrm{WGDOP} & =\sqrt{\frac{2 \cdot[(1 / \omega) \cdot(4+p)+(12+q+m+n)]}{a+c-16+2 \cdot[(4+p)+(12+q+m+n)]}} \\
& =\sqrt{\frac{2 \cdot((1 / \omega) \cdot P+G)}{(a+c-16+2 \cdot(P+G))}},
\end{aligned}
$$

where $G=Q+M+N=12+q+m+n$

Notice that $B_{12} B_{34}, B_{13} B_{24}, B_{12} B_{13} B_{23}, B_{12} B_{14} B_{24}$, $B_{13} B_{14} B_{34}, B_{23} B_{24} B_{34}, B_{12}^{2}, B_{13}^{2}, B_{14}^{2}, B_{23}^{2}, B_{24}^{2}, B_{34}^{2},(4+p)$, and $(12+q+m+n)$ all appear twice in the WGDOP 
TABLE 3: Comparison of average WGDOP residual for the proposed formulae and Rprop-based algorithm.

\begin{tabular}{lcc}
\hline & Proposed WGDOP formulae & Rprop-based algorithm \\
\hline Average WGDOP residual for Type 1 & $3.7101 * 10^{-11}$ & 0.2385 \\
Average WGDOP residual for Type 2 & $3.7062 * 10^{-11}$ & 0.2311 \\
\hline
\end{tabular}

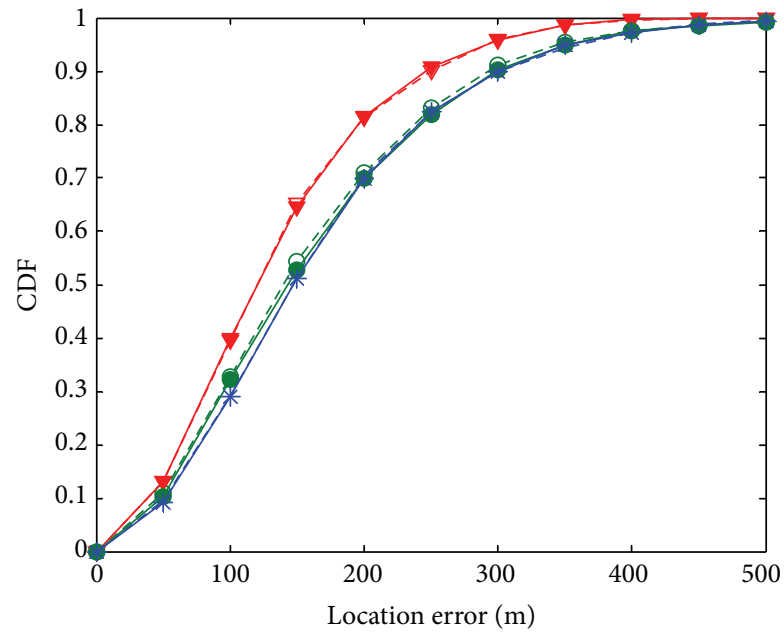

$-\nabla-$ Distance-weighted (matrix inversion)
$-\bigcirc-$ Threshold (matrix inversion)
--- LOP (matrix inversion)
$-\nabla$ Distance-weighted (proposed WGDOP formulae)
- - Threshold (proposed WGDOP formulae)
* LOP (proposed WGDOP formulae)

FIGURE 1: CDFs of the location error for various methods when four measurements have different error variances (Type 1).

express; thus sixteen multiplications and four additions can be eliminated. The value $\omega$ is also treated as a constant in the WGDOP calculation. From Table 2, this closedform solution only needs 39 multiplications (including the constant multiplication by $4,2,2$, and 2), 44 additions, 1 division, and 1 square root.

4.2.2. $2 \mathrm{D}$ Case. The WGDOP in the $2 \mathrm{D}$ case is expressed as (38). The WGDOP calculation in the $2 \mathrm{D}$ case requires 6 multiplications and 6 additions fewer than that in the 3D case. The closed-form equation needs only 33 multiplications (including the constant multiplications by 4, 2, 2, and 2), 38 additions, 1 division, and 1 square root. An alternative closedform solution of the WGDOP calculation has been presented in this paper, in which one measurement provides superior location precision over the others.

\section{Simulation Results}

Time of arrival (TOA) is major time based method and usually used in calculating the mobile station (MS) location in cellular communication systems. It is consisting of seven base stations (BSs) in cellular communication system. The serving BS and its six neighboring BSs are separated by $5 \mathrm{~km}$, and the

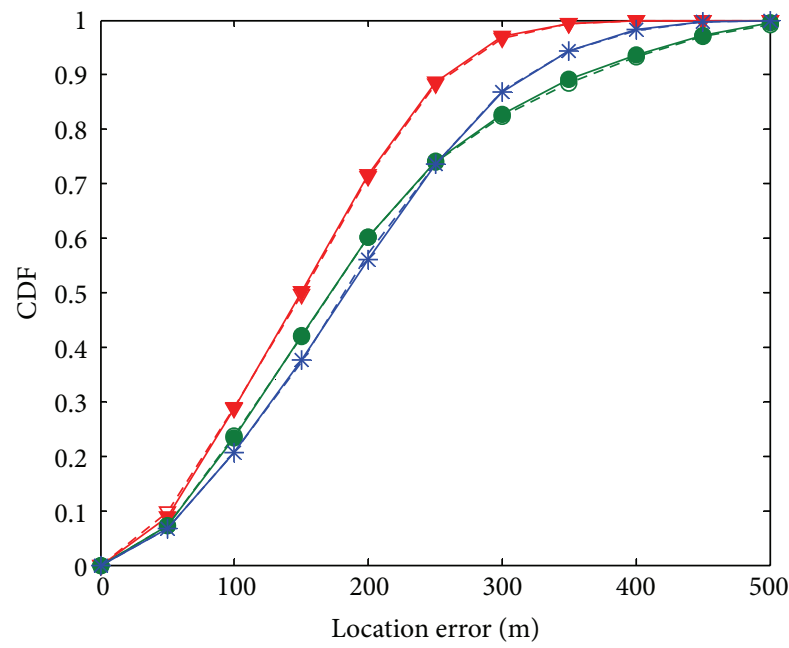

$-\nabla-$ Distance-weighted (matrix inversion)
$-\bigcirc-$ Threshold (matrix inversion)
--- LOP (matrix inversion)
- Distance-weighted (proposed WGDOP formulae)
- Threshold (proposed WGDOP formulae)
* LOP (proposed WGDOP formulae)

Figure 2: Comparison of CDFs of location error for various methods when four measurements have two types of error variances (Type 2).

MS is randomly placed among the BSs [25]. The non-line-ofsight (NLOS) propagation model is based on the uniformly distributed noise model [24], in which the TOA NLOS errors from all the BSs are different and assumed to be uniformly distributed over $\left(0, U_{i}\right)$, for $i=1,2, \ldots, 7$ where $U_{i}$ is the upper bound. For Type 1, the variables are chosen as follows: $U_{1}=200 \mathrm{~m}, U_{2}=400 \mathrm{~m}, U_{3}=350 \mathrm{~m}, U_{4}=700 \mathrm{~m}$, $U_{5}=300 \mathrm{~m}, U_{6}=500 \mathrm{~m}$, and $U_{7}=350 \mathrm{~m}$. For Type 2, the variables are given as follows: $U_{1}=200 \mathrm{~m}$ and $U_{i}=500$, for $i=2,3, \ldots, 7$. The diagonal elements of the weighted matrix $W$ are utilized with the reciprocal of the square root of an upper bound of the NLOS errors.

In order to verify the superior properties of the proposed formulae, we compare the results of WGDOP calculation accuracy for the proposed formulae and matrix inversion method. The WGDOP residual is defined as the difference between the proposed formulae and matrix inversion method. Table 3 shows average WGDOP residual for the proposed formulae and Rprop-based algorithm. For Type 1 and 2, the proposed formulae always provide much better WGDOP residual than Rprop-based algorithm [23].

We can evaluate the positioning accuracy with minimum WGDOP algorithm; MS location can be estimated by the 


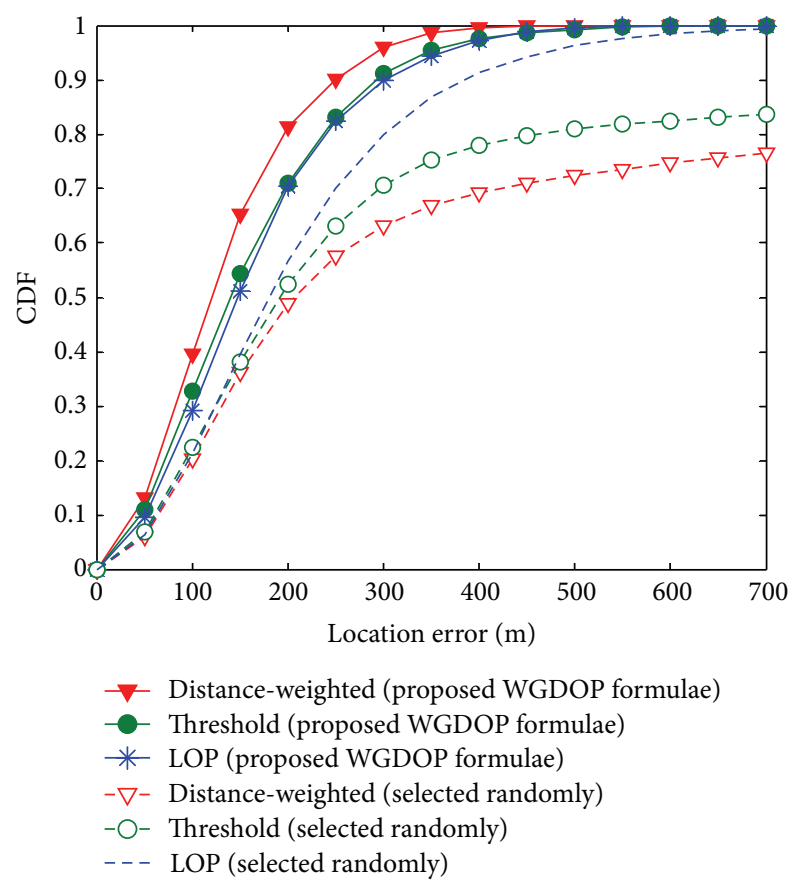

Figure 3: Comparison of location error CDFs using the subset with proposed minimum WGDOP approximation and the subset selected four BSs randomly (Type 1).

linear lines of position algorithm (LOP) [26], distanceweighted method, and threshold method which we have proposed in [27]. When four measurements have different error variances (Type 1) or four measurements have two types of error variances (Type 2), the proposed WGDOP formulae and matrix inversion method provide the nearly identical MS location estimation, as shown in Figures 1 and 2.

For Type 1, Figure 3 shows the CDFs of the average location error of these methods with different subset. Four randomly selected BSs with poor geometry perform extremely worse location estimation, and the location accuracy can be strongly affected by the relative geometry between BSs and MS. The proposed Type 2 of efficient WGDOP formulae can give better location estimation than the subsets with four BSs taken from seven BSs randomly regardless of the different methods, as shown in Figure 4. The positioning accuracy would be seriously affected by the geometric configuration of BSs and MS. In order to eliminate the poor geometry influence and improve the positioning accuracy, the selection of BSs with minimum WGDOP approximation can be used and optimal geometric configuration with four BSs is obtained.

\section{Conclusion}

To reduce the computational overhead and improve location performance, the selection of optimal measurement units is necessary. The concept of GDOP is commonly used to determine the geometric effect of GPS satellite configurations. The conventional matrix inversion method is rather time consuming and requires a great deal of computational effort. The four measurement units selected from the maximum

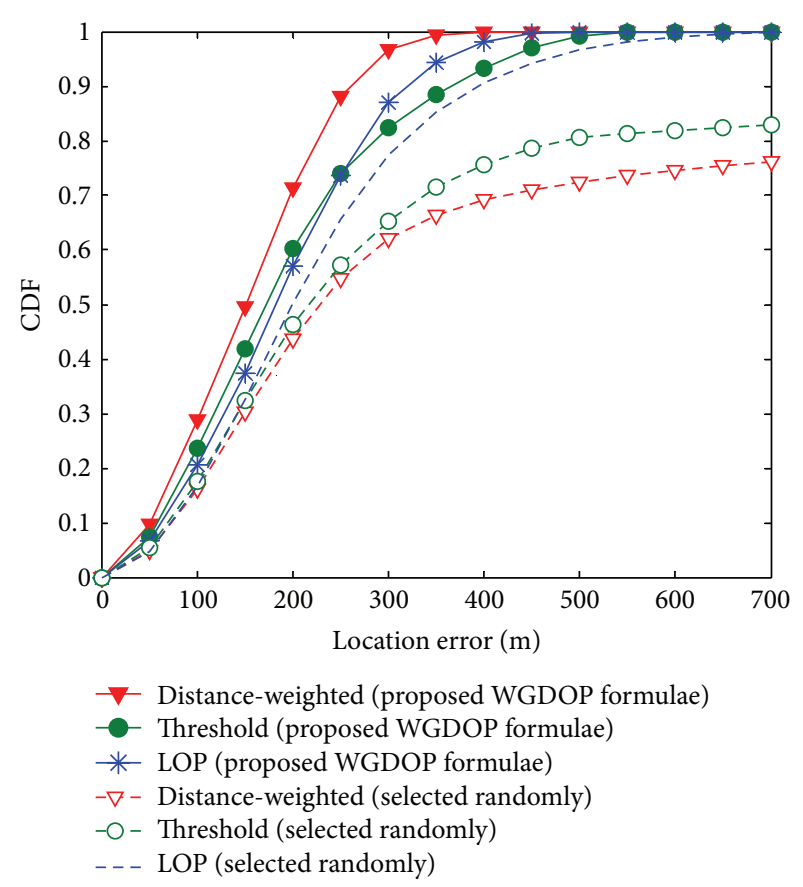

FIGURE 4: CDFs of location error of the subset with proposed minimum WGDOP formulae, and the subset selected four BSs randomly (Type 2).

volume method may not be the optimal selection. Taking into account that the variance of each measurement variance is not equal, we choose the WGDOP instead of GDOP as the criteria to select the optimal measurement units. Due to the limited power and computation capability of many mobile devices and the great number of visible satellites, to obtain WGDOP efficiently from range measurements is very critical. To further reduce the complexity, novel closed-form solutions are proposed in this paper to compute WGDOP when there are exactly four measurements available for location estimation. The efficient closed-form formulae of two formations WGDOP calculations with less effort have been proposed, in which the priori error information of each measurement is not the same. If exactly four measurements are used, the proposed formulae can provide the best computational efficiency. The proposed formulae are applicable not only to GPS but also for the WSN and cellular communication systems. The WGDOP calculations for fast evaluation are able to reduce the computational load and eliminate the poor geometry influence. The proposed efficient formulae can provide very precise solution of WGDOP calculation without incurring any approximation error.

\section{References}

[1] G. Sun, J. Chen, W. Guo, and K. J. R. Liu, "Signal processing techniques in network-aided positioning: a survey of state-ofthe-art positioning designs," IEEE Signal Processing Magazine, vol. 22, no. 4, pp. 12-23, 2005.

[2] D. Simon and H. El-Sherief, "Navigation satellite selection using neural networks," Neurocomputing, vol. 7, no. 3, pp. 247-258, 1995. 
[3] D. Simon and H. El-Sherief, "Fault-tolerant training for optimal interpolative nets," IEEE Transactions on Neural Networks, vol. 6, no. 6, pp. 1531-1535, 1995.

[4] D. E. Rumelhart, G. E. Hinton, and R. J. Williams, "Learning representations by back-propagating errors," Nature, vol. 323, no. 6088 , pp. 533-536, 1986.

[5] D.-J. Jwo and K.-P. Chin, "Applying back-propagation neural networks to GDOP approximation," Journal of Navigation, vol. 55, no. 1, pp. 97-108, 2002.

[6] C.-S. Chen and S.-L. Su, "Resilient back-propagation neural network for approximation 2-D GDOP," in Proceedings of the International MultiConference of Engineers and Computer Scientists, vol. 2, pp. 900-904, March 2010.

[7] D. Y. Hsu, "Relations between dilutions of precision and volume of the tetrahedron formed by four satellites," in Proceedings of the IEEE Position Location and Navigation Symposium, pp. 669676, April 1994.

[8] M. Kihara and T. Okada, "A satellite selection method and accuracy for the global positioning system," Navigation, vol. 31, no. 1, pp. 8-20, 1984.

[9] J. Zhu, "Calculation of geometric dilution of precision," IEEE Transactions on Aerospace and Electronic Systems, vol. 28, no. 3, pp. 893-894, 1992.

[10] E. D. Kaplan and C. J. Hegarty, Understanding GPS: Principles and Applications, Artech House Press, Boston, Mass, USA, 2006.

[11] M. Kihara, "Study of a GPS satellite selection policy to improve positioning accuracy," in Proceedings of the IEEE Position Location and Navigation Symposium, pp. 267-273, April 1994.

[12] G. M. Siouris, Aerospace Avionics Systems-A Modern Synthesis, Academic Press, San Diego, Calif, USA, 1993.

[13] C. Park, I. Kim, J. G. Lee, and G.-I. Jee, "A satellite selection criterion incorporating the effect of elevation angle in GPS positioning," Control Engineering Practice, vol. 4, no. 12, pp. 1741-1746, 1996.

[14] H. Sairo, D. Akopian, and J. Takala, "Weighted dilution of precision as quality measure in satellite positioning," IEE Proceedings, vol. 150, no. 6, pp. 430-436, 2003.

[15] Y. Yong and M. Lingjuan, "GDOP results in all-in-view positioning and in four optimum satellites positioning with GPS PRN codes ranging," in Proceedings of the Position Location and Navigation Symposium, pp. 723-727, April 2004.

[16] M. Pachter, J. Amt, and J. Raquet, "Accurate positioning using a planar pseudolite array," in Proceedings of the IEEE/ION Position, Location and Navigation Symposium, pp. 433-440, May 2008.

[17] K. Kawamura and T. Tanaka, "Study on the improvement of measurement accuracy in GPS," in Proceedings of the SICEICASE International Joint Conference, pp. 1372-1375, October 2006.

[18] B. Xu and S. Bingjun, "Satellite selection algorithm for combined gpsgalileo navigation receiver," in Proceedings of the 4th International Conference on Autonomous Robots and Agents, pp. 149-154, February 2009.

[19] C. Hongwei and S. Zhongkang, "A nonlinear optimized location algorithm for bistatic radar system," in Proceedings of the IEEE National Aerospace and Electronics Conference, vol. 1, pp. 201205, May 1995.

[20] N. Levanon, "Lowest GDOP in 2-D scenarios," IEE Proceedings, vol. 147, no. 3, pp. 149-155, 2000.
[21] H. Lu and X. Liu, "Compass augmented regional constellation optimization by a multi-objective algorithm based on decomposition and PSO," Chinese Journal of Electronics, vol. 21, no. 2, pp. 374-378, 2012.

[22] M. Zhang, J. Zhang, and Y. Qin, "Satellite selection for multiconstellation," in Proceedings of the IEEE/ION Position, Location and Navigation Symposium, pp. 1053-1059, May 2008.

[23] C.-S. Chen, J.-M. Lin, and C.-T. Lee, "Neural network for WGDOP approximation and mobile location," Mathematical Problems in Engineering, vol. 2013, Article ID 369694, 11 pages, 2013.

[24] S. Venkatraman, J. Caffery Jr., and H.-R. You, "A novel ToA location algorithm using LoS range estimation for NLoS environments," IEEE Transactions on Vehicular Technology, vol. 53, no. 5, pp. 1515-1524, 2004.

[25] J. Caffery Jr. and G. Stuber, "Subscriber location in CDMA cellular networks," IEEE Transactions on Vehicular Technology, vol. 47, pp. 406-416, 1998.

[26] J. J. Caffery Jr., "A new approach to the geometry of TOA location," in Proceedings of the 52nd Vehicular Technology Conference, pp. 1943-1949, September 2000.

[27] C.-S. Chen, S.-L. Su, and Y.-F. Huang, "Hybrid TOA/AOA geometrical positioning schemes for mobile location," IEICE Transactions on Communications, vol. E92-B, no. 2, pp. 396402, 2009. 


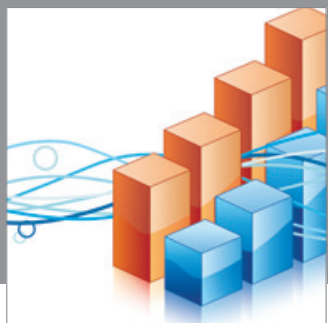

Advances in

Operations Research

mansans

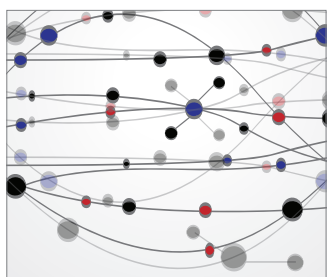

The Scientific World Journal
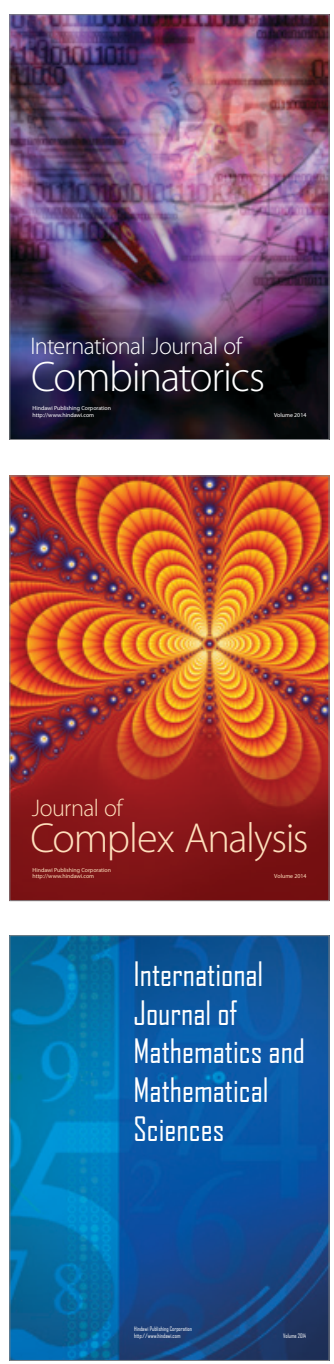
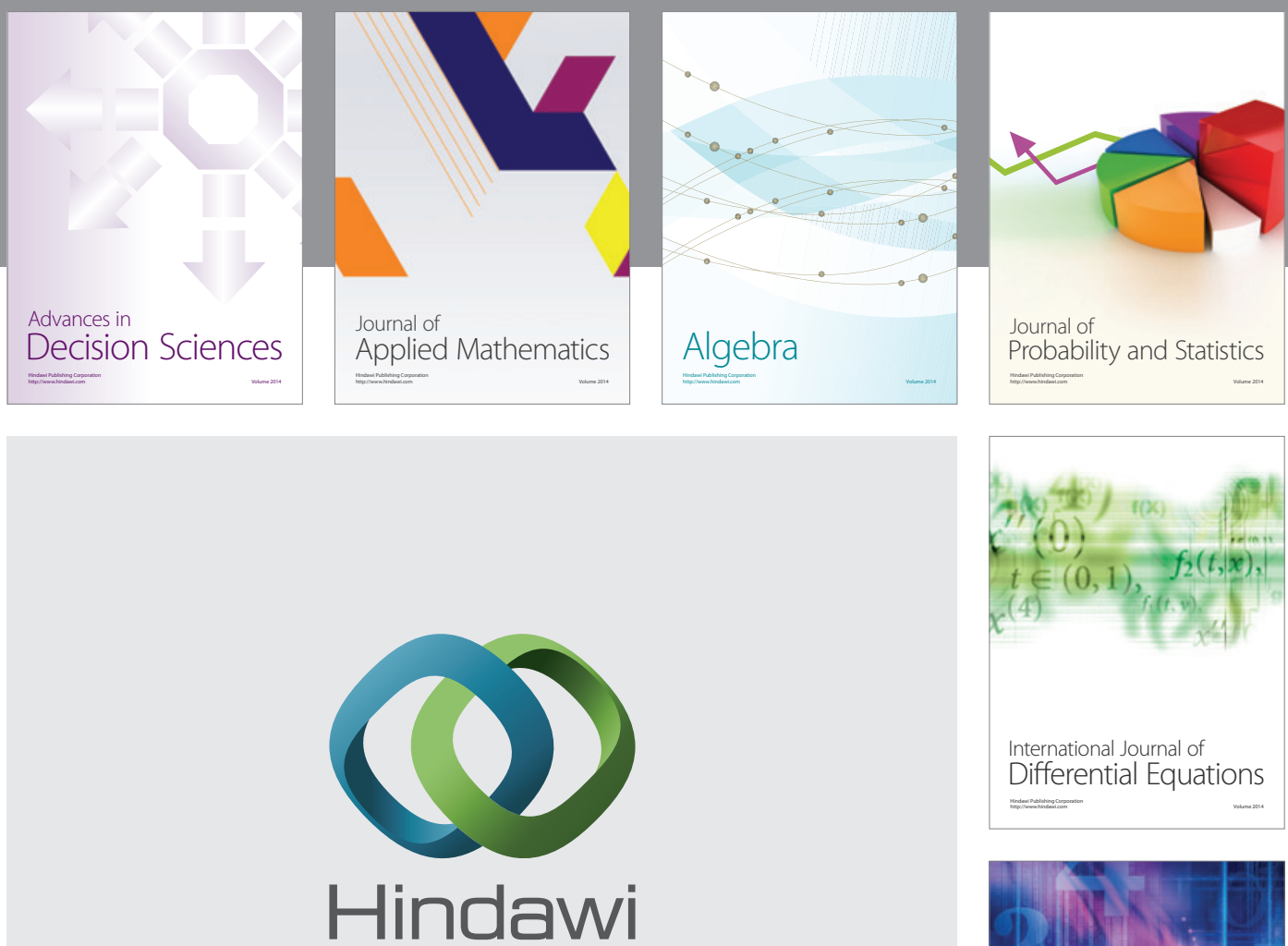

Submit your manuscripts at http://www.hindawi.com
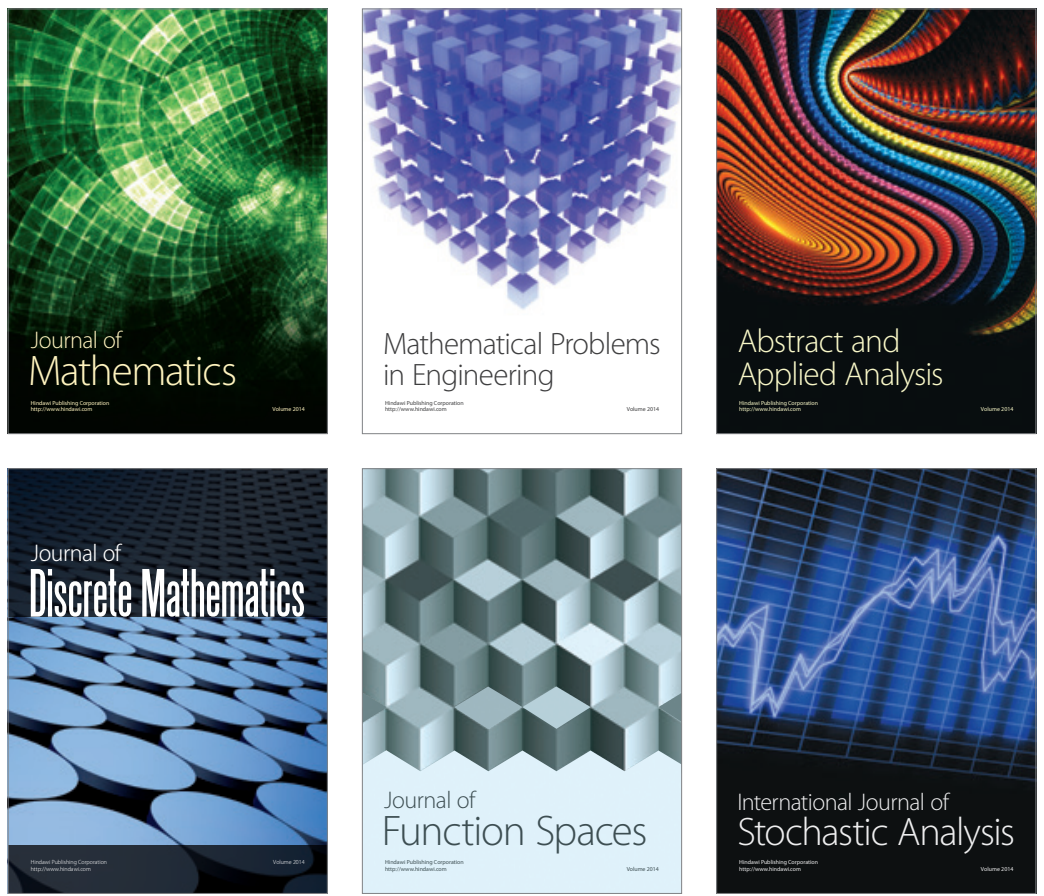

Journal of

Function Spaces

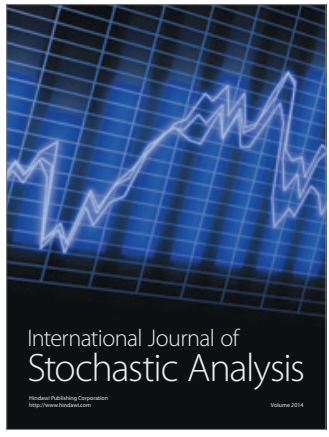

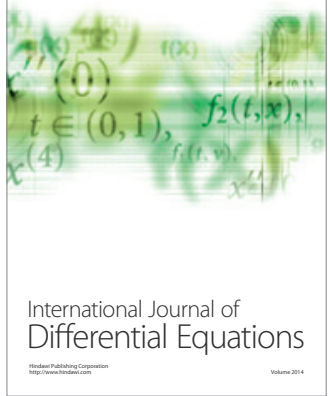
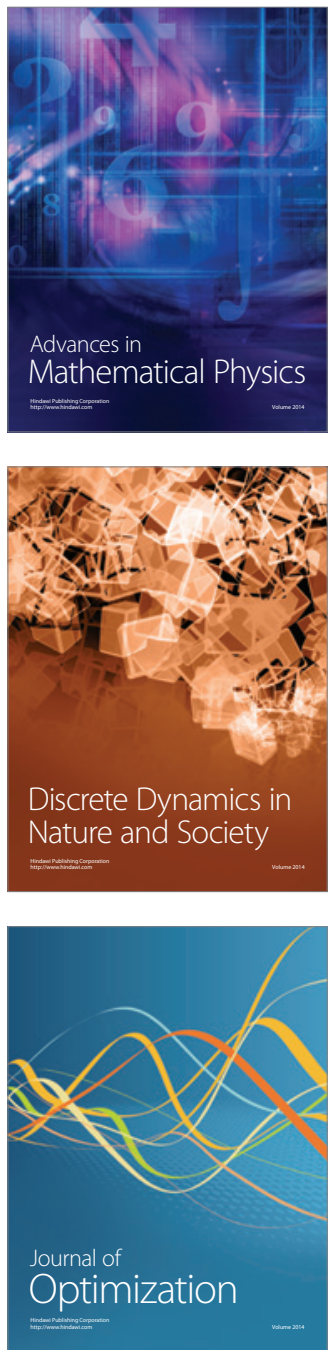